LUNG CANCER

\title{
BTS randomised feasibility study of active symptom control with or without chemotherapy in malignant pleural mesothelioma: ISRCTN 54469112
}

\author{
M F Muers, R M Rudd, M E R O'Brien, W Qian, A Hodson, M K B Parmar, D J Girling, on behalf of \\ the British Thoracic Society Mesothelioma Group*
}

Thorax 2004;59:144-148. doi: 10.1136/thorax.2003.009290

See end of article for authors' affiliations

\section{Correspondence to:} Dr W Qian, MRC Clinical Trials Unit, 222 Euston Road, London NW1 2DA, UK; wendi.qian@ctu. mrc.ac.uk

Received 29 April 2003 Accepted 12 October 2003
Background: The incidence of mesothelioma is rising rapidly in the UK. There is no generally accepted standard treatment. The BTS recommends active symptom control (ASC). It is not known whether chemotherapy in addition prolongs survival or provides worthwhile palliation with acceptable toxicity. Palliation as recorded by patients has been fully reported for only two regimens: mitomycin, vinblastine, and cisplatin (MVP), and vinorelbine (N). The BTS and collaborators planned to conduct a phase III randomised trial comparing $\mathrm{ASC}$ only, $\mathrm{ASC}+\mathrm{MVP}$, and $\mathrm{ASC}+\mathrm{N}$ in 840 patients with survival as the primary outcome measure. The aim of the present study was to assess the acceptability of the trial design to patients and the suitability of two standard quality of life (QL) questionnaires for mesothelioma.

Methods: Collaborating centres registered all new patients with mesothelioma. Those eligible and giving informed consent completed EORTC QLQ-C30+LC13 and FACT-L QL questionnaires and were randomised between all three or any two of (1) ASC only, (2) ASC+4 cycles of MVP, and (3) ASC+12 weekly doses of $\mathrm{N}$.

Results: During 1 year, 242 patients were registered of whom 109 (45\%) were randomised $155 \%$ of the 197 eligible patients). Fifty two patients from 20 centres were randomised to an option including ASC only. This translates into a rate of 312 per year from 60 centres interested in collaborating in the phase III trial. The EORTC QL questionnaire was superior to FACT-L in terms of completeness of data and patient preference. Clinically relevant palliation was achieved with ASC.

Conclusion: The planned phase III trial is feasible.
M alignant mesothelioma is almost invariably fatal and its incidence is rising rapidly. In the UK the age standardised death rate per 100000 rose from 0.33 in 1970-4 to 1.20 in 1990-4, and it is estimated that the annual number of deaths from mesothelioma will rise from approximately 1500 in the year 2000 to a peak of approximately 3000 in 2020. ${ }^{12}$ The highest incidence is seen in men born in 1945-50, reflecting the extent of use of asbestos in the 1960s and 1970s at the beginning of their working lives.

There is no generally agreed standard treatment for unresectable mesothelioma. According to BTS recommendations at the time the present study was planned and conducted, patients should be treated with "active symptom control" (ASC) involving: (1) regular specialist follow up, (2) structured assessment of physical, psychological and social problems with appropriate treatment, which can include pleurodesis, palliative radiotherapy and steroids, (3) rapid referral to additional specialists when required, and (4) parallel nursing support. ${ }^{3}$

Clinicians disagree on whether any anticancer chemotherapy prolongs survival or provides worthwhile palliation with acceptable toxicity when given in addition to ASC. Numerous small non-randomised studies of various single drug and multidrug regimens have shown that a number have activity against mesothelioma, and a review of studies involving 15 or more patients made the following observations: ${ }^{4}$

- The role of systemic chemotherapy should be regarded as an area of investigation.

*Members of the British Thoracic Society Mesothelioma Group are listed at the end of the paper.
- Various single agents have shown temporary partial response rates of around $20 \%$, although response is difficult to measure in mesothelioma because of the diffuse nature of this tumour.

- There is no persuasive evidence that drug combinations are more active than single drugs.

- Randomised trials are needed to confirm activity, to investigate clinical usefulness, and to determine whether responses translate into prolonged survival.

Response rates do not necessarily reflect symptom relief, which is more important for patients with an incurable disease. At the time when this study was planned, good data on symptom control from patients' questionnaires were available for only two regimens. Middleton and colleagues treated 39 patients with six cycles of mitomycin, vinblastine and cisplatin (MVP) repeated every 21 days. ${ }^{5}$ In all, $62 \%$ of patients had an overall improvement in their symptoms, palliation being particularly good for pain $(79 \%)$ and cough $(67 \%)$. Palliation was achieved in all of the $20 \%$ of patients showing a partial tumour response, but worthwhile symptom control was also seen in some of those without such response. The regimen was well tolerated. Steele and colleagues treated 29 patients with single drug vinorelbine $(\mathrm{N})$ every 7 days until disease progression. ${ }^{6}$ Improvement in respiratory symptoms was reported by $48 \%$ of patients and in psychological functioning by $76 \%$. The regimen was well tolerated.

In a systematic review of the literature, trial databases, colleagues, and the pharmaceutical industry, we found three published randomised trials, ${ }^{7-9}$ but all three were small (32, 76, and 79 patients, respectively) and compared one chemotherapy regimen with another. There were three more 
closed but as yet unpublished randomised trials. One (EST1380) compared postoperative radiotherapy with or without doxorubicin; the second (NCI-93-0204C) debulking surgery + chemo-immunotherapy with or without intrapleural photodynamic therapy; and the third (Lilly Oncology, NCIG00-1767) cisplatin with or without multitargeted folate antagonist (pemetrexed) in 456 patients. Thus, no other randomised trial has included an ASC only group.

A large randomised phase III trial is needed to assess whether, in the treatment of malignant pleural mesothelioma, chemotherapy with MVP or N in addition to ASC is better than ASC alone in terms of overall survival, symptom palliation, performance status, analgesic usage, toxicity, quality of life (QL), tumour response, and progression-free survival. Before embarking on such a trial we conducted the present feasibility study to assess the acceptability of the randomisation to patients and the suitability of the EORTC QLQ-C30+LC13 and the FACT-L QL questionnaires in mesothelioma.

\section{METHODS}

\section{Patients}

In collaborating centres all new patients with a diagnosis of mesothelioma were registered with the MRC Clinical Trials Unit. Registered patients were eligible for randomisation if they fulfilled the following criteria:

- microscopically and immunohistochemically confirmed malignant pleural mesothelioma, including epithelial and other histological types;

- any symptomatic pleural effusion under control by drainage, pleurodesis, or pleurectomy;

- a CT scan within 1 month before randomisation (preferably after pleurodesis);

- if mesothelioma resected, two CT scans 6 weeks apart showing assessable stable or progressive disease;

- no previous chemotherapy for mesothelioma;

- no other disease likely to interfere with protocol treatments or comparisons;

- WHO performance status $0-2 ;^{10}$

- white blood cell count $>3 \times 10^{9} / \mathrm{l}$, neutrophil count $>1.5$ $\times 10^{9} / \mathrm{l}$, platelet count $>100 \times 10^{9} / \mathrm{l}$, and no clinical evidence of infection;

- medically fit to receive chemotherapy;

- informed consent form signed following full discussion of a patient information sheet describing the study design and stating that the doctor would discuss with the patient which comparison was most suitable;

- quality of life forms completed before patient told the treatment allocated.

Multicentre Research Ethics Committees (MREC) approval of the protocol was obtained. Confirmation of Local Research Ethics Committee's (LREC) approval was required before a centre could start registering patients.

\section{Treatment groups}

Clinicians were encouraged to randomise eligible patients between all three treatment groups: (1) ASC only; (2) ASC+MVP; (3) ASC $+\mathrm{N}$, but were allowed to offer patients randomisation between any two. This choice was permitted to ensure a good measure of the acceptability of the ASC only arm to both patients and clinicians.

\section{Active symptom control (ASC)}

The essential elements were as follows:

- Regular follow up in a specialist clinic by an identified physician or team.
- Structured assessments at every clinic visit of physical, psychological, and social problems with appropriate treatment or other action.

- Rapid involvement of additional specialists such as a pain relief service, specialist palliative care team, medical social worker, or physiotherapist. (It was recommended that patients with pain not easily controlled by a combination of slow release morphine and co-analgesics be referred to a pain relief service.)

- Parallel nursing support from a named specialist nurse or similar person.

ASC could include treatment with palliative radiotherapy and steroids.

Mitomycin, vinblastine, and cisplatin (MVP)

Mitomycin $8 \mathrm{mg} / \mathrm{m}^{2}$ by bolus intravenous injection, vinblastine $6 \mathrm{mg} / \mathrm{m}^{2}$ (maximum dose $10 \mathrm{mg}$ ) by bolus intravenous injection, and cisplatin $50 \mathrm{mg} / \mathrm{m}^{2}$ by intravenous infusion over 4 hours were given every 21 days for a total of four cycles with standard hydration and anti-emetics.

\section{Vinorelbine (N)}

Six intravenous injections at weekly intervals followed by a 2 week break before a further six injections at weekly intervals of vinorelbine $30 \mathrm{mg} / \mathrm{m}^{2}$ (maximum dose $60 \mathrm{mg}$ ) were given, with standard anti-emetics.

\section{QL questionnaires}

All patients completed EORTC QLQ-C30+LC13 ${ }^{11}$ and FACT-L $\mathrm{L}^{12}$ QL questionnaires. To avoid bias that may arise if one questionnaire is always completed before the other, the sequence in which they were completed was randomised by centre, the sequence remaining the same for every assessment of all patients from that centre.

\section{Assessments}

Pretreatment assessments included a CT scan of the thorax; the QL questionnaires had to be completed before the patients were told their treatment group. During the treatment period, when MVP and N patients were receiving chemotherapy, all patients were assessed clinically every 3 weeks. Thereafter, patients were assessed every 8 weeks until death. QL questionnaires were completed immediately before all assessments. A CT scan was performed at the end of chemotherapy or 3-4 months after randomisation in the ASC only group.

\section{Statistical methods}

The outcome measures were the acceptability of the study design to patients, suitability of the EORTC QLQ-C30+LC13 and FACT-L QL questionnaires for mesothelioma, and palliation. Acceptability of the design was measured by the proportions of registered patients accepting randomisation and of randomised patients accepting ASC only as one of their randomisation options. The suitability of the QL questionnaires was assessed by compliance with their use and patient preference: patients were asked to state their preferred QL questionnaire but were not asked their reasons. Palliation was measured in terms of WHO performance status $^{10}$ and severity of chest pain, breathlessness, anorexia, and sweating (present "not at all" =0, "a little" =1, "quite a bit" $=2$, or "very much" $=3$ ). Palliation was defined as: (1) improvement (change from a score of 2 or 3 to 0 or 1), (2) control (a score of 1 not getting worse), and (3) prevention (a score of 0 being maintained) ${ }^{13}$ from baseline to 16 weeks after randomisation, based on clinicians' assessments. The study was not powered to compare tumour response or survival between treatment groups; these outcome measures 
are therefore not reported but are included in the phase III trial.

\section{RESULTS}

\section{Acceptability of the trial design}

From September 2000 to September 2001, 242 patients were registered from 46 UK centres. Of the 197 eligible for randomisation, 109 from 35 centres were randomised, 55\% (95\% CI 48 to 62 ) of those eligible and $45 \%$ of those registered. The main reasons for non-randomisation were ineligibility and patient refusal (table 1).

The randomisation options are shown in table 2. A third of patients were randomised to the recommended option (between all three treatment groups), but the option included ASC only in $52(48 \%)$ of the 109 patients (95\% CI 38 to 57 ), these 52 being randomised from 20 centres. With an eventual rate of randomisation including ASC only of 8.7 patients per month (104 per year), this translates into an estimated rate of $312(104 \times 3)$ per year from the 60 centres that expressed an interest in collaborating in the phase III trial.

The characteristics of the patients at registration or randomisation according to their randomisation group are shown in table 3. The three groups of patients (randomised with ASC only option, randomised without ASC only option, not randomised) were very similar except that there was a tendency for the group randomised without the ASC only option to be younger than the group with this option (median age 61 and 66 years, respectively).

\section{Comparison of the two QL questionnaires}

Compliance with the completion of the QL questionnaires was similar (table 4) but with a higher rate of missing responses per question with the FACT-L (9.1\%) than with the EORTC QLQ-C30+LC13 (6.9\%). Questionnaire preference was expressed on 386 forms. Although on 291 (75\%) occasions patients expressed no preference between the questionnaires, on 75 occasions (19\%) they preferred the EORTC QLQC30+LC13 questionnaire and on 20 occasions (5\%) the FACT$\mathrm{L}$ questionnaire was preferred.

\section{Palliation of symptoms}

The analysis of palliation of symptoms was based on patients assessed before treatment and with more than one subsequent assessment during the first 16 weeks after randomisation, or who died during the 16 weeks but with at least one assessment after randomisation. Patients treated with ASC only were compared with those treated with ASC plus any chemotherapy (table 5). There is a suggestion that palliation was better with chemotherapy than without, but there is evidence that clinically useful levels of palliation were achieved with ASC only $(28 \%$ for chest pain, $11 \%$ for breathlessness, $44 \%$ for anorexia, and $67 \%$ for sweating attacks). It is important to note, however, that the study was not powered to make reliable comparisons between treatment groups. All the patients had WHO performance status

\begin{tabular}{|c|c|}
\hline Reason & Patients, $\mathbf{n}\left(\%^{*}\right)$ \\
\hline Not eligible & $45(34)$ \\
\hline Eligible, patient refusal & $47(36)$ \\
\hline Clinician's decision & $29(22)$ \\
\hline Other & $11(8)$ \\
\hline Reason not known & 1 \\
\hline Total not randomised & 133 \\
\hline
\end{tabular}

*Percentage of the 132 patients for whom the reason is known.
Table 2 Randomisation options for all 109 randomised patients

\begin{tabular}{ll}
\hline Options & Patients, $\mathbf{n}(\%)$ \\
\hline ASC versus MVP versus N & $35(32)$ \\
ASC versus MVP & $2(2)$ \\
ASC versus N & $15(14)$ \\
MVP versus N & $57(52)$ \\
All those including ASC only & $52(48)$ \\
\hline
\end{tabular}

$\mathrm{ASC}=$ active symptom control; $\mathrm{MVP}=$ mitomycin, vinblastine and cisplatin; $\mathrm{N}=$ vinorelbine.

$\mathrm{ASC}=\mathrm{ASC}$ only; $\mathrm{MVP}=\mathrm{ASC}+\mathrm{MVP} ; \mathrm{N}=\mathrm{ASC}+\mathrm{N}$.

grades 0,1 , or 2 before treatment. In both patient groups these levels changed very little during the first 16 weeks (details not shown).

\section{DISCUSSION}

This feasibility study has shown that, in the treatment of malignant pleural mesothelioma, randomisation to ASC with or without chemotherapy was sufficiently acceptable to patients and clinicians for a large multicentre randomised trial comparing ASC only versus ASC+MVP versus ASC $+\mathrm{N}$ to be feasible. Of the patients eligible for randomisation, 55\% were randomised between all three or any two of the treatment groups, half of whom accepted a randomisation option including ASC only. Indeed, intake to a multicentre cancer research UK trial (MSOl) in which all patients are being randomised between all three treatment groups is now underway and is currently attracting support from 52 centres. At the time we made our grant application for the phase III trial, 60 centres that expressed an interest estimated that between them they could randomise more than 400 patients per year. Allowing for overestimation, we plan to recruit a total of 840 patients (280 per treatment group) during 4 years, including those already randomised between the three treatment groups in the present feasibility study. The current mean rate of accrual is three patients per centre per year.

Crucial to the study of the possible roles of chemotherapy in the treatment of mesothelioma, a cancer relatively resistant to chemotherapy, is the study of quality of life as reported by patients. Only in this way can the possible benefits and adverse effects be adequately assessed. An important finding from the present feasibility study is therefore the suitability of the EORTC QLQ-C30+LC13 questionnaire for use in patients with pleural mesothelioma, and this instrument is being used in the subsequent MSOl phase III trial.

The study was not powered to make reliable comparisons between the treatment groups, but an important finding is that clinically relevant levels of palliation were achieved with ASC only. It is therefore important to establish whether chemotherapy improves palliation compared with ASC.

After the intake to the MSOl trial had started, results of the randomised trial comparing cisplatin with or without pemetrexed (ALIMTA) in 456 patients were presented at the 2002 Annual Conference of the American Society of Clinical Oncology and appeared to show a survival advantage for pemetrexed + cisplatin over cisplatin alone. ${ }^{14}$ The question therefore arose whether intake to the MS0l trial should continue. We decided that it should for the following reasons. Firstly, the pemetrexed trial has only been presented and the findings were not included in the published abstract; details of its design, methods, analyses, and findings are therefore not yet publicly available and have not been peer reviewed. Secondly, pemetrexed is not yet available in the UK and is unlikely to be so for at least 2 years. Thirdly, the data presented showed that the pemetrexed trial design was 


\begin{tabular}{|c|c|c|c|}
\hline \multirow[b]{2}{*}{ Characteristic } & \multicolumn{2}{|l|}{ Randomised } & \multirow[b]{2}{*}{$\begin{array}{l}\text { Not randomised } \\
\text { n (\%) }\end{array}$} \\
\hline & $\begin{array}{l}\text { With ASC only } \\
\text { option, } n(\%)\end{array}$ & $\begin{array}{l}\text { Without ASC only } \\
\text { option, } n(\%)\end{array}$ & \\
\hline \multicolumn{4}{|l|}{ Age } \\
\hline$<60$ & $13(25)$ & $24(42)$ & $29(22)$ \\
\hline $60-69$ & 19 (37) & $26(46)$ & $46(35)$ \\
\hline $70+$ & $20(39)$ & 7 (12) & 58 (44) \\
\hline Median (range) & $66(48-80)$ & $61(38-78)$ & $69(43-87)$ \\
\hline \multicolumn{4}{|l|}{ Sex } \\
\hline Male & $47(90)$ & $46(81)$ & $111(83)$ \\
\hline Female & $5(10)$ & 11 (19) & $22(17)$ \\
\hline \multicolumn{4}{|l|}{ Histology } \\
\hline Epithelial & $40(77)$ & $43(75)$ & NA \\
\hline \multirow{2}{*}{\multicolumn{4}{|c|}{ Performance status }} \\
\hline & & & \\
\hline 0 & $5(10)$ & $8(14)$ & NA \\
\hline 1 & $33(64)$ & $35(61)$ & NA \\
\hline 2 & $14(27)$ & $14(25)$ & NA \\
\hline Total patients & 52 & 57 & 133 \\
\hline
\end{tabular}

Table 4 Compliance with completion of quality of life questionnaires

\begin{tabular}{lll}
\hline Compliance & $\begin{array}{l}\text { EORTC QLQ- } \\
\text { C30+LC13 }\end{array}$ & FACT-L \\
\hline Number of questionnaires expected & 798 & 798 \\
Number of questionnaires received & 578 & 584 \\
Number of questions per questionnaire & 44 & 38 \\
Number of missing items & 1747 & 2024 \\
Mean number of missing items per & 3.0 & 3.5 \\
questionnaire & $6.9 \%$ & $9.1 \%$ \\
Mean missing rate per question & & \\
\hline
\end{tabular}

changed during patient recruitment because of high toxicity and death rates in the pemetrexed arm. Full vitamin B 12 and folate supplementation was introduced part way through for all subsequent patients. This reduced the toxicity and death rates and the planned number of patients was increased. The survival difference in the fully vitamin supplemented patients was of borderline significance $(p=0.051)$ and eight patients were excluded from this analysis (that is, it was not done by intention to treat). Fourthly, the median age was lower (61 years) and the performance status of the patients higher (only ECOG 0-1 eligible) than those typically seen in patients in the UK and, indeed, in the current feasibility study. It is therefore uncertain whether similar results would be achievable in UK practice. Finally, it is questionable whether single drug cisplatin, in a moderately high dose $\left(75 \mathrm{mg} / \mathrm{m}^{2}\right)$ for up to six cycles, is an appropriate control: it achieved, as expected, a low response rate ( $17 \%$ according to nonstandard criteria); it gave rise to $6 \%$ grade 3 or 4 vomiting and $2 \%$ drug related deaths; and it was associated with increasing pain and dyspnoea during treatment. It could well be that, at least in terms of quality of life, ASC as given in the present study is superior, especially in the light of the palliation it achieved. ${ }^{15}$ A recent systematic review of published phase II studies concluded that the role of chemotherapy for unresectable malignant mesothelioma is unclear but that the combination of cisplatin and doxorubicin should be considered as a control for randomised

Table 5 Palliation of symptoms during the first 16 weeks after randomisation*

\begin{tabular}{|c|c|c|c|c|}
\hline \multirow[b]{2}{*}{ Nature of palliation $†$} & \multicolumn{2}{|l|}{ ASC only } & \multicolumn{2}{|c|}{ ASC plus chemotherapy } \\
\hline & Assessable & Palliated & Assessable & Palliated \\
\hline \multicolumn{5}{|l|}{ Chest pain } \\
\hline Improvement & 9 & 2 & 22 & 12 \\
\hline Control & 7 & 2 & 26 & 16 \\
\hline Prevention & 2 & 1 & 36 & 16 \\
\hline Total palliated & 18 & $5(28 \%)$ & 84 & $44(52 \%)$ \\
\hline \multicolumn{5}{|l|}{ Breathlessness } \\
\hline Improvement & 7 & 0 & 24 & 7 \\
\hline Control & 9 & 1 & 29 & 14 \\
\hline Prevention & 2 & 1 & 16 & 2 \\
\hline Total palliated & 18 & $2(11 \%)$ & 83 & $23(28 \%)$ \\
\hline \multicolumn{5}{|l|}{ Anorexia } \\
\hline Improvement & 4 & 0 & 12 & 7 \\
\hline Control & 4 & 4 & 11 & 6 \\
\hline Prevention & 10 & 4 & 61 & 30 \\
\hline Total palliated & 18 & $8(44 \%)$ & 84 & $43(51 \%)$ \\
\hline \multicolumn{5}{|l|}{ Sweating attacks } \\
\hline Improvement & 4 & 2 & 18 & 9 \\
\hline Control & 3 & 3 & 19 & 12 \\
\hline Prevention & 11 & 7 & 47 & 32 \\
\hline Total palliated & 18 & $12(67 \%)$ & 84 & $53(63 \%)$ \\
\hline
\end{tabular}

*This analysis was based on clinicians' assessments from which more data were available than from the questionnaires, but the results are very similar to those from the patient questionnaires. †Defined in the text. 
trials. ${ }^{16}$ This recommendation was, however, based solely on response rates.

Confidential interim analyses from the MS01 trial will continue to be regularly reviewed by its independent data monitoring and ethics committee. Moreover, this committee is also reviewing results of the pemetrexed trial as they emerge and can recommend changes to the MS0l protocol if and when it considers these to be ethically desirable.

\section{Authors' affiliations}

M F Muers, General Infirmary, Leeds LS1 3EX, UK

R M Rudd, St Bartholomew's Hospital, London EC1A 7BE, UK

M E R O'Brien, Royal Marsden Hospital, Sutton, Surrey SM2 5PT, UK W Qian, A Hodson, M K B Parmar, D J Girling, MRC Clinical Trials Unit, London NWI 2DA, UK

The trial was sponsored by The June Hancock and Anthony Farmer Mesothelioma Research Funds, Pierre Fabre Oncology, and the British Thoracic Society.

\section{Members of the BTS Mesothelioma Group \\ Chairman: M F Muers, Leeds General Infirmary.}

Clinical members: M Britton, St Peter's Hospital, Chertsey; C M B Higgs, Dorothy House Hospice, Bradford-on-Avon; M Lind, The Princess Royal Hospital, Hull; R Milroy, Stobhill NHS Trust, Glasgow; M Nicolson, Aberdeen Royal Infirmary, Aberdeen; M E R O'Brien, Royal Marsden Hospital, Sutton; M Peake, Glenfield Hospital, Leicester; N Rowell, Royal Marsden Hospital, Sutton; R M Rudd, St Bartholomew's Hospital, London; I E Smith, Royal Marsden Hospital, Sutton; J P C Steele, St Bartholomew's Hospital, London.

Patient advocacy: M Robinson, Patient Mesothelioma Information Line, Cookridge Hospital, Leeds.

Medical Research Council Clinical Trials Unit staff: D J Girling, M K B Parmar, W Qian, MRC Clinical Trials Unit, 222 Euston Road, London NW1 2DA.

The following consultants and their colleagues entered patients: Bath Royal United Hospital (E D Gilby, A S Malin); Birmingham Heartlands Hospital (D R Ferry); Bristol Oncology Centre (S J Falk, M Tomlinson); Cambridge Addenbrooke's and Papworth Hospitals (D Gilligan, S Old); Chelmsford Broomfield Hospital (N Davidson); Cheltenham General Hospital (D Farrugia, P J Jenkins); Clatterbridge Centre for Oncology (J Littler, E Marshall, J Maguire, S O'Reilly, A J Slater, A McGuire, J E S Brock, S Myint); Darlington Memorial Hospital (E N Evans); Derbyshire Royal Infirmary (D Guthrie, R B Kulkarni); Dundee Ninewells Hospital (E Rankin); Durham Dryburn Hospital (N Munro); Edinburgh Western General Hospital (A Price); Exeter Royal Devon and Exeter Hospital ( $P$ Bliss); Hartlepool (P Sutton); Glan Clwyd Hospital, North Wales (A E Champion); Inverness Raigmore Hospital (D Whillis); Ipswich Hospital (J $S$ Morgan); Leeds General Infirmary and Cookridge Hospitals (M G Bond, M F Muers, D O'Riordan, M P Snee); Leicester Royal Infirmary (K J O'Byrne); Lincoln County Hospital (L D Nuortio); Llandough Hospital and Velindre Hospital (F R Macbeth); London Guy's and St Thomas' Cancer
Centre and Hammersmith Hospital (P A Ellis, P G Harper); Maidstone Mid-Kent Oncology Centre/Royal Marsden (M E R O'Brien); Manchester Wythenshawe Hospital (H Anderson, N Thatcher); Middlesborough James Cook University Hospital/Northallerton Friarage Hospital (H R Gribbin, J C M Van Der Voet); Newport Chest Clinic (G Williamson); Newport Royal Gwent Hospital (A E Brewster); Peterborough District General Hospital (K Fife); Plymouth Derriford Hospital (C R McGavin); Sheffield Weston Park Hospital (K Dunn, P M Fisher, M Hatton, P Kirkbride, P Lorrigan); Southampton Royal South Hants Hospital (P M W Johnson, A Last, C Ottensmeier); Southend General Hospital (A Lamont, C W L Trask); Stoke-on-Trent North Staffordshire Royal Infirmary (A Cook); Sunderland Royal Infirmary (H Clague); Swansea Singleton Hospital (K Rowley); Torbay Hospital (J M Goldman); Walsall Manor Hospital (A D Chetiyawardana, R Joshi); Wolverhampton New Cross Hospital (C Brammer); York District Hospital (A M Hunter).

The Clinical Trials Managers were D Lobban, D Andrews, A Hodson, and R Owens.

\section{REFERENCES}

1 Peto J, Hodgson JT, Matthews FE, et al. Continuing increase in mesothelioma mortality in Britain. Lancet 1995;345:535-9.

2 Peto J, Decarli A, La Vecchia C, et al. The European mesothelioma epidemic. $\mathrm{Br} J$ Cancer 1999;79:666-72.

3 British Thoracic Society Standards of Care Committee. Statement on the diagnosis and management of mesothelioma. Thorax 2001;56:250-65.

4 Ryan CW, Herndon J, Vogelzang NJ. A review of chemotherapy trials for malignant mesothelioma. Chest 1998;113:66S-73S.

5 Middleton GW, Smith IE, O'Brien MER, et al. Good symptom relief with palliative MVP (mitomycin-C, vinblastine and cisplatin) chemotherapy in malignant mesothelioma. Ann Oncol 1998;9:269-73.

6 Steele JPC, Shamash L, Evans MT, et al. Phase II study of vinorelbine in patients with malignant pleural mesothelioma. J Clin Oncol 2000;18:3912-7.

7 Sorensen PG, Bach F, Bork E, et al. Randomised trial of doxorubicin versus cyclophosphamide in diffuse malignant pleural mesothelioma. Cancer Treat Rep 1985;69:1431-2.

8 Samson MK, Wasser LP, Borden EC, et al. Randomized comparison of cyclophosphamide, imidazole carboxamide, and adriamycin versus cyclophosphamide and adriamycin in patients with advanced stage malignant mesothelioma: a Sarcoma Intergroup Study. J Clin Oncol 1987;5:86-91.

9 Chahinian AP, Antman K, Goutsou M, et al. Randomized phase II trial of cisplatin with mitomycin or doxorubicin for malignant mesothelioma by the Cancer and Leukemia Group B. J Clin Oncol 1993;11:1559-65.

10 World Health Organization. WHO handbook for reporting results of cancer treatment. WHO Offset Publication No. 48. Geneva: WHO, 1979.

11 Fayers P, Aaronson N, Bjordal K, et al. EORTC QLQ-C30 scoring manual. Brussels: EORTC Study Group on Quality of Life, 1999.

12 Cella DF. FACT manual, Version 3. Chicago: Luke's Medical Center, 1996

13 Stephens RJ, Hopwood P, Girling DJ. Defining and analysing symptom palliation in cancer clinical trials: a deceptively difficult exercise. $\mathrm{Br} J$ Cancer 1999;79:538-44.

14 Vogelzang NJ, Rusthoven J, Paoletti P, et al. Phase III single-blinded study of pemetrexed + cisplatin vs. cisplatin alone in chemonaive patients with malignant pleural mesothelioma. Proc Am Soc Clin Oncol 2002;21:2a, abstract 5 (available at http://virtualmeeting.asco.org/vm2002/default.cfm select 'lectures' then 'general oncology' then 'plenary session' then talk by Vogelzang.).

15 Steele JPC. The new front line treatment for malignant pleural mesothelioma? Thorax 2003;58:96-7.

16 Berghmans T, Paesmans M, Lalami Y, et al. Activity of chemotherapy and immunotherapy on malignant mesothelioma: a systematic review of the literature with meta-analysis. Lung Cancer 2002;38:111-21. 